\title{
ÉDITORIAL
}

\section{Bienvenue à RP Magazine}

La revue a changé. Je vous avais annoncé dans mon premier éditorial qu'elle changerait encore : parlons en dans ce premier numéro de 1997.

Radioprotection a toujours été une pièce essentielle dans la vie de la Société Française de Radioprotection mais elle se distingue, par rapport aux revues d'autres sociétés, par son contenu, mélange d'articles scientifiques et techniques et d'informations. L'équilibre entre ces deux composantes est difficile à trouver et à maintenir. Il permet à nos lecteurs de mieux participer à la vie de notre société.

Durant l'année 1996, nous avons longuement discuté du rôle de notre revue et de sa forme, tant au sein du Comité de rédaction qu'au sein du Conseil d'administration, sous l'impulsion vigoureuse de notre présidente Annie Sugier.

Plusieurs questions se sont posées, mais la principale était sans aucun doute : fallait-il publier séparément la partie scientifique et technique et la partie informations; ou fallait-il maintenir ces deux parties dans une seule et même publication tout en les différenciant? La réponse apparaît dans ce premier numéro de l'année 1997.

Dans sa première partie, notre revue continuera de publier les articles scientifiques et techniques revus par des lecteurs anonymes, je tiens à le rappeler. Ces articles continueront à paraître sous les rubriques classiques : articles de synthèse, articles, notes techniques, produits nouveaux ; puis viendront les revues des livres et le courrier des lecteurs qui traite de questions scientifiques et techniques. Vous y verrez dans un prochain numéro des articles « invités ", où une personnalité reconnue traitera d'un sujet scientifique ou technique ayant ou pouvant avoir une répercussion importante sur les pratiques de radioprotection. Le changement se fait en douceur, j'espère qu'il n'en est pas moins perceptible. Continuez à nous envoyer vos articles car c'est grâce à vous que nous atteindrons les objectifs de qualité que nous nous sommes fixés.

En revanche, nous bouleversons la partie Informations, ce qui explique le retard dans la parution de ce premier numéro, mais je suis sur que vous nous en excuserez.

La Société Francaise de Radioprotection

a le plaisir de vous annoncer la naissance de

$R P$ Magazine 
Ce titre, vous ne pourrez l'oublier : il figurera en tête de toutes les rubriques. RP Magazine a sa structure propre, son sommaire et ses rubriques. La Lettre de la Présidente y trouvera tout naturellement sa place en ouverture; nous avons pensé que les Entretiens, que vous semblez apprécier, devaient figurer en bonne place; viennent ensuite les rubriques classiques concernant notre société ainsi que les nouvelles plus générales. Ces rubriques bien ordonnées seront repérables aisément par la répétition de leur nom dans un bandeau grisé qui bordera toutes les pages du magazine. La police de caractères est différente : nous voulons que vous puissiez naviguer sans ambiguïté dans la revue et selon votre choix commencer par la partie scientifique ou la partie magazine. Ce magazine sera coordonné par notre secrétaire général, Jacques Lombard, membre ex-officio du Comité de rédaction. Il sera aidé par notre nouveau Président émérite Daniel Blanc.

D'autres points sont encore en discussion : il s'agit d'un agrandissement du format qui permettrait de traiter RP Magazine sur deux colonnes, avec pour conséquence une présentation plus moderne et la possibilité d'insérer plus facilement des photos, et de la parution de ce magazine sur des pages de couleur avec pour corollaire un plus grand cout. Ces changements, s'ils interviennent, ne se feront pas avant 1998 car nous voulions tout d'abord que RP Magazine naisse et recueille votre avis.

J'espère que vous apprécierez ces changements, et je serais très heureux de connaître vos commentaires sur l'ensemble de la revue et tout particulièrement sur notre tout nouveau RP Magazine. J'attends également des suggestions car la revue est l'un des reflets tangibles du dynamisme de notre société.

Vous noterez que le changement n'a pas eu lieu qu'en France : nos membres correspondants étrangers changent en partie ; je tiens à remercier les «partants », pour tous les services qu'ils ont rendus, et je souhaite la bienvenue à Christer Viktorsson, Mounir Besbes, HU Zunsu qui sont les premiers représentants d'une nouvelle vague de correspondants étrangers et qui nous aideront à mieux faire connaître encore Radioprotection en Suède, en Tunisie et en Chine.

Ces mutations auraient été difficiles à négocier si je ne pouvais compter sur un Comité de rédaction solide et sur l'arrivée à mes cotés de Christian Lefaure, Viceprésident du Comité de rédaction. C'est donc une équipe unie, bien renouvelée, qui aborde l'année 1997 et les changements qu'elle apportera.

Avant de conclure, j'aimerais au nom du Comité de rédaction remercier pour tous les services rendus à notre revue, Jeanne Berger, qui après avoir dirigé avec efficacité les Éditions de Physique, notre éditeur, a pris une retraite que je souhaite la plus heureuse possible. C'est donc avec Jean-Marc Quilbé, le nouveau Directeur à qui le Comité présente tous ses vœux de réussite que nous relèverons les défis du changement.

H. MÉTIVIER

Président du Comité de la Revue 